\title{
Detection of extrapulmonary lesions with integrated PET/CT in the staging of
} lung cancer

\author{
W. De Wever*, Y. Vankan*, S. Stroobants ${ }^{\#}$ and J. Verschakelen*
}

ABSTRACT: The aim of the present study was to assess retrospectively the additional value of positron emission tomography (PET)/computed tomography (CT) in the detection of unexpected extrapulmonary lesions in the staging of patients with a malignant pulmonary lesion in comparison with CT and PET used alone.

A total of 217 patients with a pathologically proven lung tumour underwent PET/CT. CT, PET and PET/CT were evaluated in the detection of extrapulmonary lesions. These abnormalities were compared with the final diagnosis obtained from the medical records and statistical analysis was carried out.

In total, 108 lesions were clinically detected. PET/CT showed a sensitivity, specificity, positive and negative predictive values and accuracy of $100,81,71,100$ and $87 \%$, respectively, for the detection of extrapulmonary lesions and $92,98,89,98$ and $97 \%$, respectively, for the detection of malignant extrapulmonary lesions. PET/CT was significantly better than CT and PET used alone.

Conventional staging work-up has a poor sensitivity in detecting second primary cancers or unexpected metastases. The detection of malignant extrapulmonary lesions is necessary for correct tumour staging. By combining both metabolic and anatomical information, positron emission tomography/computed tomography is able to depict more unexpected extrapulmonary lesions than computed tomography and positron emission tomography used alone, and positron emission tomography/computed tomography provides more additional information of malignancy or benignancy of lesions detected with one of the two imaging modalities alone.

KEYWORDS: Extrapulmonary lesion, integrated positron emission tomography/computed tomography, lung cancer

ung cancer is a common disease and is the leading cause of death in many countries.

Several imaging techniques are available to detect distant metastases of lung cancer. Computed tomography (CT) is the routine imaging procedure for staging patients with lung cancer in many institutions. Usually, a combination of a CT scan of the brain to exclude brain metastasis, a CT scan of the upper abdomen to exclude liver and adrenal metastasis, and bone scintigraphy to exclude bone metastases are performed [1]. However, the technique of CT is often not able to exclude reliably extrapulmonary metastases, especially occult metastases, and to detect second primary cancers. Many reports suggest that F-fluoro-2-deoxyglucose positron emission tomography (FDG-PET) is more sensitive than $\mathrm{CT}$ in the diagnosis of extrapulmonary lesions [2]. FDG-PET can detect unsuspected occult metastases and second primary cancers.
In the study by STROOBANTS et al. [3], an additional detection of distant malignant lesions was found in $5 \%$ of patients. According to STROOBANTS et al. [3], the most important contribution of FDG-PET was its ability to exclude malignancy in the most distant lesions using equivocal CT.

Recently, integrated PET/CT has been introduced more routinely into clinical situations [4]. Integrated PET/CT enables the direct correlation of FDG-accumulating lesions with morphological structures. It has been shown that integrated PET/ $\mathrm{CT}$ improves $\mathrm{T}$ and $\mathrm{N}$ staging of nonsmall cell lung cancer in comparison with other imaging methods [5]. The clinical significance of extrapulmonary focal abnormality on FDG-PET often remains unclear, given that FDG accumulation is not specific for malignant tissue [6]. FDG accumulation may reflect metastatic disease, but can also reflect an additional pathology unrelated to the lung
AFFILIATIONS

Depts of *Radiology, and

\# Nuclear Medicine, University Hospitals Gasthuisberg

Leuven, Belgium.

CORRESPONDENCE

W. De Wever

Dept of Radiology

University Hospitals Gasthuisberg

Herestraat 49

3000 Leuven

Belgium

Fax: 3216343765

E-mail: walter.dewever@uz.

kuleuven.ac.be

Received:

September 122006

Accepted after revision:

February 082007

STATEMENT OF INTEREST

None declared.

European Respiratory Journal Print ISSN 0903-1936 Online ISSN 1399-3003 
cancer for which the patient was originally referred [7]. Initial literature reports have shown that the precise localisation of hypermetabolic lesions by PET/CT may change the definition of focal intra-abdominal FDG uptake from indeterminate or equivocal to benign aetiology, and may therefore improve the diagnostic accuracy of FDG-PET [8].

The aim of the present study was to assess retrospectively the additional value of integrated PET/CT in the detection of unexpected extrapulmonary lesions in the staging of patients with a suggestive or proven malignant pulmonary lesion (in particular, those lesions that could be missed by the classical staging procedures [1]) in comparison with CT and PET used alone.

\section{PATIENTS AND METHODS}

\section{Patient population}

Between June 2003 and August 2005, 400 consecutive patients (309 male and 91 female, mean age 57 yrs) underwent an integrated PET/CT for staging of a suggestive lung lesion. Of these 400 patients, 307 had an anatomopathological evaluation of the lesion (43 benign lesions, 264 malignant lesions). The aetiology of the different lesions is summarised in table 1 .

The 217 patients with a proven malignant lung tumour underwent integrated PET/CT with intravenous contrast administration. Only these patients were finally included in the present retrospective study.

The clinical judgment concerning an additional extrapulmonary lesion was made by the physicians, who generally considered the lesion to be suggestive of metastasis, an additional primary tumour or an additional nontumoural lesion on the basis of anatomopathology, comparison with previous examinations or follow-up. The aetiology of the extrapulmonary lesions is summarised in table 2.

\begin{tabular}{|c|c|c|}
\hline TABLE 1 & $\begin{array}{l}\text { Anatomopatholo } \\
\text { of the primary lu }\end{array}$ & ution \\
\hline \multicolumn{3}{|c|}{ Malignant tumour } \\
\hline \multicolumn{2}{|c|}{ Squamous cell carcinoma } & 110 \\
\hline \multicolumn{2}{|c|}{ Adenocarcinoma } & 96 \\
\hline \multicolumn{2}{|c|}{ Large cell carcinoma } & 21 \\
\hline \multicolumn{2}{|c|}{ Small cell carcinoma } & 21 \\
\hline \multicolumn{2}{|c|}{ Adenoid cystic carcinoma } & 1 \\
\hline \multicolumn{2}{|c|}{ Spindle cell carcinoma } & 1 \\
\hline \multicolumn{2}{|c|}{ Thymic carcinoma } & 1 \\
\hline \multicolumn{2}{|c|}{ Carcinoid tumour } & 4 \\
\hline \multicolumn{2}{|c|}{ EBV-associated myogenic tumour } & 2 \\
\hline \multicolumn{2}{|c|}{ Malignant mesothelioma } & 3 \\
\hline \multicolumn{2}{|c|}{ Metastases } & 4 \\
\hline \multicolumn{3}{|c|}{ Benign tumour } \\
\hline \multicolumn{2}{|c|}{ Hamartoma } & 2 \\
\hline \multicolumn{2}{|c|}{ Vascular malformation } & 2 \\
\hline \multicolumn{2}{|c|}{ Infection } & 11 \\
\hline \multicolumn{2}{|c|}{ Normal or sequellar lesion } & 28 \\
\hline
\end{tabular}

Data are presented as n. EBV: Epstein-Barr virus.

\section{Imaging protocol}

All patients were examined on a dual-modality PET/CT tomograph (Biograph LSO duo; Siemens Medical Solutions Inc., Hoffman Estates, IL, USA). The CT component of the Biograph LSO duo corresponds to a Somatom Emotion Duo (Siemens Medical Solutions Inc.), a two-row spiral CT system with a maximum continuous scan time of $100 \mathrm{~s}$ and a maximum rotation speed of 75 r.p.m. CT images were acquired at $85 \mathrm{~mA}, 130 \mathrm{kV}$, a slice thickness of $5 \mathrm{~mm}$ and a table feed of $12 \mathrm{~mm}$ per rotation. The scanning area for CT and PET was defined on a CT topogram. Single-section, wholebody spiral CT was performed, starting with the head and subsequently covering the neck, thorax, abdomen and pelvis. To ensure diagnostic CT image quality, $120 \mathrm{~mL}$ of a contrast agent containing $300 \mathrm{mg}$ of iodine per millilitre (Xenetix 300; Guerbet, Sulzbach, Germany) was administered either intravenously using an automated injector $\left(1.6 \mathrm{~mL} \cdot \mathrm{s}^{-1}\right.$, scan delay $100 \mathrm{~s}$ ) or orally to fill the gastro-intestinal tract (GIT). A limited breath-hold technique was used to avoid motion-induced artefacts in the area of the diaphragm.

The PET component of the combined PET/CT tomograph is based on an ECAT ACCEL (Siemens Medical Solutions Inc.), a full-ring lutetium orthosilicate-based PET system with an inplane spatial resolution of $4.6 \mathrm{~mm}$ and a transverse field of view of $15.5 \mathrm{~cm}$ for each bed position. PET images were corrected for attenuation on the basis of the CT data, and iterative reconstruction algorithms with two iterations and eight subsets were performed. PET imaging was performed $75 \mathrm{~min}$ after the administration of $4.5 \mathrm{MBq} \cdot \mathrm{kg}^{-1}$ of FDG. Patients had been instructed to fast for a minimum of $4 \mathrm{~h}$ prior to starting the examination. Blood samples collected before the injection of the radioactive tracer ensured blood glucose levels were within the normal range.

\section{Data analysis}

CT images

CT evaluation was performed using the CT images obtained from the integrated PET/CT scanner. A chest radiologist interpreted these CT images whilst blinded to the information from the PET part of the integrated PET/CT; the only information available was that the integrated PET/CT had been carried out to stage a suggestive lung lesion. The radiologist was asked to determine if there were extrapulmonary lesions on the CT scan and to make a decision about the nature of these lesions. Based on CT characteristics, an extrapulmonary lesion can either be suggestive of a malignant lesion (either a metastatic lesion or second primary cancer) or it can have the characteristics of a benign lesion. In the present study, a lesion that is very suggestive of malignancy based on its imaging characteristics has been defined as an imaging-based malignant lesion (iML); a lesion that is suggestive of a benign lesion on imaging is defined as an imaging-based benign lesion (iBL).

\section{PET images}

PET evaluation was performed by a nuclear medicine physician who interpreted the PET part of the integrated PET/CT without knowledge of the information from CT. Only the information that an integrated PET/CT was performed to stage a suggestive lung lesion was available. The nuclear medicine physician was also asked to determine if extrapulmonary lesions were 


\begin{tabular}{|c|c|c|}
\hline Site of lesion & Aetiology of lesion & $\mathbf{n}$ \\
\hline \multicolumn{3}{|c|}{ Expected sites ${ }^{\#}$} \\
\hline \multirow[t]{3}{*}{$\mathrm{iBL}$} & Enlargement of the adrenal gland or discrete FDG uptake & 18 \\
\hline & Lesion in the liver & 22 \\
\hline & Bone alteration & 1 \\
\hline \multirow[t]{4}{*}{$\mathrm{iML}$} & Adrenal metastases & 13 \\
\hline & Liver metastases & 8 \\
\hline & Brain metastases & 4 \\
\hline & Bone metastases & 8 \\
\hline \multicolumn{3}{|c|}{ Unexpected sites } \\
\hline \multirow[t]{9}{*}{$\mathrm{iBL}$} & Gastro-intestinal tract & 3 \\
\hline & Kidney & 1 \\
\hline & Thyroid & 1 \\
\hline & Soft tissue & 2 \\
\hline & Bladder & 1 \\
\hline & Pancreas & 2 \\
\hline & Prostate & 1 \\
\hline & Organomegaly & 1 \\
\hline & Enlarged lymphadenopathies & 13 \\
\hline \multirow[t]{5}{*}{$\mathrm{iML}$} & Primary intestinal tumour & 2 \\
\hline & Kidney tumour & 2 \\
\hline & Soft tissue tumour & 1 \\
\hline & Peritoneal metastases & 2 \\
\hline & Metastasis in the spinal channel & 1 \\
\hline
\end{tabular}

iBL: imaging-based benign lesion; iML: imaging-based malignant lesion; FDG: F-fluoro-2-deoxyglucose. * : Expected sites include the liver, adrenal glands, brain and bone (skull and upper abdomen).

present. Qualitative analysis of the images was performed by visual identification of areas of increased FDG uptake. A focally increased FDG activity above normal physiological levels was considered abnormal and to display potential malignancy. As the present study was retrospective, no surface uptake values were measured.

\section{Integrated PET/CT images}

Integrated PET/CT evaluation was performed using the fusion images produced by the integrated PET/CT scanner. A radiologist and a nuclear medicine physician in consensus evaluated the integrated PET/CT images to determine whether extrapulmonary lesions were present. When there was a discordance between $\mathrm{CT}$ and PET, the following criteria were used to decide whether the lesion was suggestive or not. 1) Lesions in the liver and in the brain suspicious for metastases on one of the two examinations were considered as positive for metastases; 2) FDG-PET-positive lesions in the abdomen not related to the liver or adrenal glands were correlated with the corresponding structure on CT to decide if they were metastatic or not; and 3) an enlarged adrenal gland on CT but negative on PET was considered not to be tumourally invaded.

\section{Statistical analysis}

Evaluation of the presence of iBL and iML with CT alone, PET alone and integrated PET/CT was performed and compared with the clinical findings based on anatomopathological evaluation, in comparison with previous examinations or on follow-up. The relative accuracies of the different imaging techniques were compared using McNemar's exact test. Sensitivities, specificities, predictive values and accuracies were calculated with respect to their ability to detect clinically relevant extrapulmonary lesions.

\section{RESULTS}

In the present study population, 108 extrapulmonary lesions (67 $(62 \%)$ benign and $41(38 \%)$ malignant lesions) were clinically retained in $69(32 \%)$ patients.

CT, PET and PET/CT retained 145 (63 iBL, 38 iML, 44 indeterminate), 64 (18 iBL, $35 \mathrm{iML}, 10$ indeterminate) and 149 (63 iBL, 41 iML, 44 indeterminate) lesions, respectively. CT missed three iML, PET and PET/CT missed six and zero iML, respectively. CT, PET and PET/CT showed 44, 10 and 44 indeterminate lesions, respectively. More details of the nature and distribution of these lesions are shown in tables 3 and 4 .

The present authors clinically found $33(30 \%)$ extrapulmonary lesions $(25(23 \%)$ benign lesions and eight (1\%) malignant lesions) at the expected sites for metastases of lung cancer (i.e. adrenal gland, liver, brain and bone metastases) [9]. CT, PET and PET/CT detected 34 (22 iBL, six iML and six indeterminate), 27 (14 iBL, eight iML and five indeterminate) and 39 (22 iBL, eight iML and nine indeterminate) lesions, respectively. More details classifying the extrapulmonary lesions further as lesions of the GIT and other lesions are given in tables 2, 3 and 4 . 
TABLE 3 Detection of extrapulmonary lesions with different imaging modalities shown according to lesions in expected and unexpected sites for metastases of a lung tumour ${ }^{\#}$

\begin{tabular}{|c|c|c|c|c|c|c|c|}
\hline \multicolumn{4}{|c|}{ Expected sites } & \multicolumn{4}{|c|}{ Unexpected sites } \\
\hline $\begin{array}{l}\text { Lesions retained } \\
\text { clinically }\end{array}$ & CT & PET & $\mathrm{PET} / \mathrm{CT}$ & $\begin{array}{l}\text { Lesions retained } \\
\text { clinically }\end{array}$ & CT & PET & PET/CT \\
\hline
\end{tabular}

\begin{tabular}{|c|c|c|c|c|c|c|c|c|}
\hline Subjects n & 75 & 111 & 36 & 109 & 33 & 34 & 27 & 39 \\
\hline ML & 33 & 32 & 27 & 33 & 8 & 6 & 8 & 8 \\
\hline$B L$ & 42 & 41 & 4 & 41 & 25 & 22 & 14 & 22 \\
\hline Indeterminate lesion & & 38 & 5 & 35 & & 6 & 5 & 9 \\
\hline ML not visualised & & 1 & 6 & 0 & & 2 & 0 & 0 \\
\hline BL not visualised & & 1 & 35 & 1 & & 3 & 11 & 3 \\
\hline
\end{tabular}

Data are presented as n. CT: computed tomography; PET: positron emission tomography; ML: malignant lesion; BL: benign lesion. ${ }^{\#}:$ total subjects, $n=108 ;{ }^{\bullet}:$ expected sites include the liver, adrenal glands, brain and bone (skull and upper abdomen).

The specificity, sensitivity, predictive values and accuracy for the detection of extrapulmonary lesions and the detection of malignant extrapulmonary lesions are summarised in table 5. The relative accuracies of the different imaging techniques for the detection of extrapulmonary lesions were compared using McNemar's exact test and are summarised in table 6 .

\section{DISCUSSION}

Conventional staging work-up has a poor sensitivity (only $50 \%$ ) in detecting a second primary tumour or unexpected metastases [10]. In the present study's patient population, 108 extrapulmonary lesions were retained within 69 patients.

PET/CT showed three $(2.8 \%$ of the lesions, $1.4 \%$ of the patients) additional malignant extrapulmonary lesions in comparison with $\mathrm{CT}$ alone and six $(5.6 \%$ of the lesions and $2.8 \%$ of the patients) in comparison with PET alone. PET/CT missed no malignant extrapulmonary lesion (c.f. CT alone missed four (fig. 1) and PET alone missed six malignant lesions). However, PET/CT could not reduce the number of

\section{TABLE 4 Distribution of detected extrapulmonary lesions}

\begin{tabular}{|c|c|c|c|c|c|c|}
\hline & Subjects $n$ & ML & BL & Indeterminate lesions & ML not detected & BL not detected \\
\hline \multicolumn{7}{|c|}{ Adrenal glands } \\
\hline CT & 48 & 13 & 18 & 17 & 0 & 0 \\
\hline PET & 12 & 10 & 0 & 2 & 3 & 18 \\
\hline PET/CT & 47 & 13 & 18 & 16 & 0 & 0 \\
\hline \multicolumn{7}{|l|}{ Liver } \\
\hline CT & 50 & 8 & 22 & 20 & 0 & 0 \\
\hline PET & 10 & 7 & 2 & 1 & 1 & 17 \\
\hline PET/CT & 47 & 8 & 21 & 17 & 0 & 1 \\
\hline \multicolumn{7}{|l|}{ Bone } \\
\hline CT & 7 & 7 & 0 & 0 & 1 & 1 \\
\hline PET & 9 & 7 & 1 & 1 & 1 & 0 \\
\hline $\mathrm{PET} / \mathrm{CT}$ & 10 & 8 & 1 & 1 & 0 & 0 \\
\hline \multicolumn{7}{|l|}{ Brain } \\
\hline CT & 6 & 4 & 1 & 1 & 0 & 0 \\
\hline PET & 5 & 3 & 1 & 1 & 1 & 0 \\
\hline PET/CT & 6 & 4 & 1 & 1 & 0 & 0 \\
\hline \multicolumn{7}{|l|}{ GIT } \\
\hline CT & 2 & 1 & 1 & 0 & 1 & 2 \\
\hline PET & 5 & 2 & 2 & 1 & 0 & 1 \\
\hline PET/CT & 6 & 2 & 3 & 1 & 0 & 0 \\
\hline \multicolumn{7}{|c|}{ Miscellaneous } \\
\hline $\mathrm{CT}$ & 32 & 5 & 21 & 6 & 1 & 1 \\
\hline PET & 23 & 6 & 12 & 4 & 0 & 10 \\
\hline PET/CT & 33 & 6 & 19 & 8 & 0 & 3 \\
\hline
\end{tabular}

Data are presented as n. ML: malignant lesion; BL: benign lesion; CT: computed tomography; PET: positron emission tomography; GIT: gastro-intestinal tract. 


\begin{tabular}{|c|c|c|c|c|c|c|}
\hline \multirow[t]{3}{*}{ TABLE 5} & \multicolumn{6}{|c|}{$\begin{array}{l}\text { Specificity, sensitivity, positive and negative } \\
\text { predictive values, and accuracy for the detection } \\
\text { of extrapulmonary lesions and for the detection } \\
\text { of image-based malignant extrapulmonary } \\
\text { lesions (iML) alone }\end{array}$} \\
\hline & \multicolumn{2}{|c|}{ СT } & \multicolumn{2}{|c|}{ PET } & \multicolumn{2}{|c|}{ РET/CT } \\
\hline & $\begin{array}{c}\mathrm{iBL} \text { and } \\
\mathrm{iML}\end{array}$ & $\mathrm{iML}$ & $\begin{array}{c}\text { iBL and } \\
\text { iML }\end{array}$ & $\mathrm{iML}$ & $\begin{array}{c}\text { iBL and } \\
\text { iML }\end{array}$ & iML \\
\hline Specificity & 80 & 98 & 92 & 98 & 81 & 98 \\
\hline Sensitivity & 91 & 18 & 47 & 22 & 100 & 92 \\
\hline PPV & 68 & 71 & 74 & 75 & 71 & 89 \\
\hline NPV & 95 & 89 & 79 & 89 & 100 & 98 \\
\hline Accuracy & 83 & 88 & 78 & 89 & 87 & 97 \\
\hline
\end{tabular}

Data are presented as \%. CT: computed tomography; PET: positron emission tomography; iBL: image-based benign lesions; PPV: positive predictive value NPV: negative predictive value.

indeterminate lesions. These results demonstrate that integrated PET/CT is a useful tool with which to screen for extrapulmonary malignant lesions (a second primary cancer or occult metastases). The present authors' findings are in the same range as those of other studies. ISHIMORI et al. [11] found a prevalence of $1.2 \%$ on PET/CT of pathology-proven additional primary malignancy. Other studies have shown a detection of additional malignancy in $1.1-8.5 \%$ of cases using PET and PET/CT [12-16]. The most important advantage of PET/CT is the possibility of an exact correlation of images with metabolic information (PET images) and images with anatomical information (CT images). PET/CT can clarify the precise location of an FDG-avid focus and, in this way, it can reduce the probability of false-positive or false-negative results. Metastases of a lung tumour can also have an unusual distribution pattern. In the conventional staging procedure, a CT of the abdomen, liver and adrenal glands is normally not performed and, thus, any unusual metastases or additional primary tumours is not detected. Integrated PET/CT can provide a solution to this problem.

In the present study and based on PET/CT imaging, 44 lesions were not clinically retained. The reason for this is not always

\begin{tabular}{|c|c|c|}
\hline \multirow[t]{3}{*}{ TABLE 6} & \multicolumn{2}{|c|}{$\begin{array}{l}\text { Relative accuracies of the different imaging } \\
\text { techniques in the detection of extrapulmonary } \\
\text { lesions compared by McNemar's exact test ( } 95 \% \\
\text { confidence interval) }\end{array}$} \\
\hline & \multicolumn{2}{|c|}{ PET/CT } \\
\hline & Extrapulmonary lesions & $\begin{array}{c}\text { Extrapulmonary malignant } \\
\text { lesions }\end{array}$ \\
\hline Ст & $p=0.2673$ & $p<0.0001$ \\
\hline PET & $p<0.0001$ & $p=0.0001$ \\
\hline
\end{tabular}

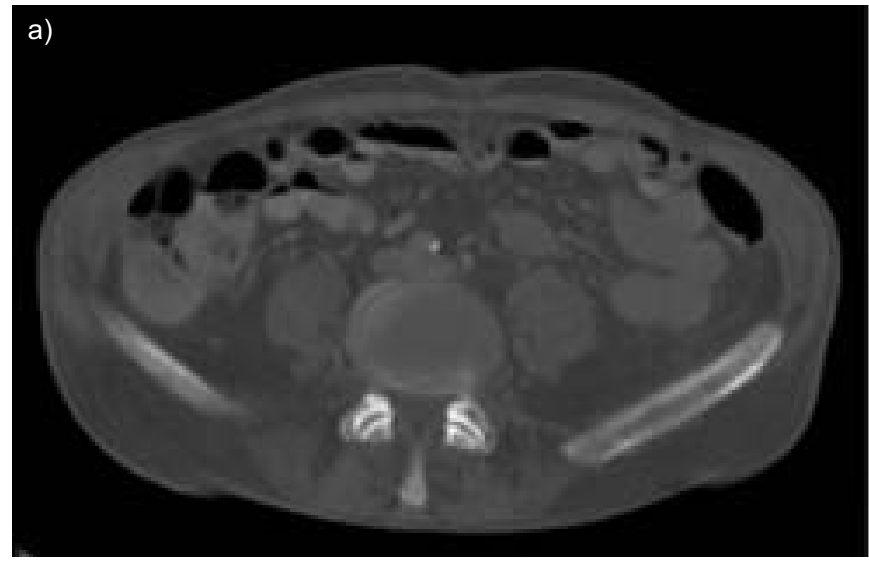

b)

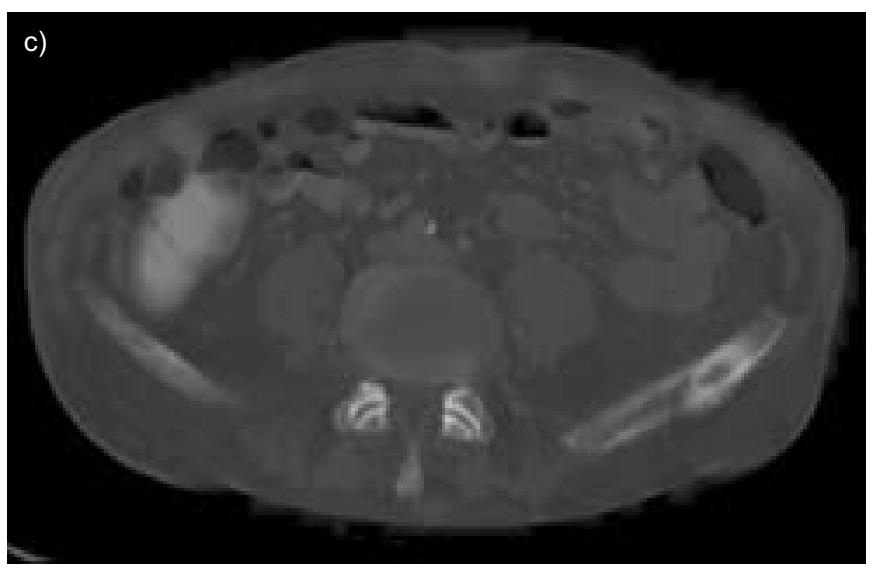

FIGURE 1. A 70-yr-old male with small cell lung carcinoma in the left lower lobe. a) The computed tomography (CT) part of the positron emission tomography (PET)/CT does not reveal the extrapulmonary lesion in the left lower abdomen. b) The PET part of the PET/CT shows an F-fluoro-2-deoxyglucose hotspot in this region. c) PET/CT demonstrates a pathological lesion into the left pelvis corresponding to a bone metastasis. This was a suggestive lesion that was out of the region scanned by the classical CT of the chest and upper abdomen.

very clear. As can be seen in table 4, most of the indeterminate lesions were situated in the liver and adrenal glands. Most of these lesions were small but must be noted, especially because they were situated in sites typical for metastases of a lung tumour. It can also be seen from table 4 that most of the time, $\mathrm{CT}$ is the imaging modality that detects these abnormalities. Due to the small size of these abnormalities, these lesions can 
appear as false negatives on PET. At the same time, this is also the reason why no further clinical investigation is carried out immediately and that follow-up is chosen for these lesions. As follow-up is still continuing in the present study, it was impossible to decide whether or not these lesions were benign and so these lesions were indicated as indeterminate. It is probable that PET information showing no suspicious lesions is correct in these cases, but further follow-up is required to prove this.

The number of indeterminate lesions on PET/CT and CT in the present study is the same; however, as can be seen in table 4 , this is probably accidental. Lesions are also detected by PET that have remained indeterminate to date, predominantly because follow-up is still on-going.

Through the high quality of its images, CT shows many small indeterminate lesions that are probably too small to detect with PET. In such cases, follow-up seems to be the only option available to avoid further unnecessary investigation.

In the patient population of the present study, 33 lesions $(15 \%$ of the patients) were detected from the expected sites of metastases of a lung tumour. PET/CT and PET detected all the malignant lesions, and CT missed two malignant lesions. Using PET/CT imaging, LARDINOIS et al. [9] found solitary extrapulmonary lesions without clearly defined clinical symptoms in $72(21 \%)$ out of 350 patients, with a high incidence of lesions unrelated to the primary lung tumour.

In the present study, five ( $2 \%$ of the patients, $5 \%$ of the lesions) lesions of the GIT were found: two were malignant lesions and three benign. CT detected one malignant and one benign lesion; PET detected two malignant lesions and missed one benign lesion; and PET/CT detected all malignant and benign lesions. ISRAEL et al. [17] found an incidental foci of abnormal FDG uptake precisely localised by PET/CT to the GIT in 1.3\% of their study population. The present study's results of detection of lesions related to the GIT are in the same range. However, there was only a small number of GIT lesions. Most gastro-intestinal tumours show a highly increased accumulation of FDG [18], but it is well known that FDG may also accumulate in some inflammatory and benign disorders of the GIT [19], and that FDG uptake of variable intensity and localisation patterns can also be caused by physiological tracer activity attributed to uptake by smooth muscles (mainly in the bowel), swallowed secretions, or excretion and intraluminal concentration of FDG (fig. 2) [20]. PET using FDG is more accurate than $\mathrm{CT}$ or other conventional imaging modalities for diagnosis of previously unknown, recurrent or metastatic malignant foci $[16,21,22]$. The additional value of PET/CT is the better localisation of FDG hotspots related to the GIT on CT and has been advocated as a useful, novel imaging tool leading to a decrease in the number of false-positive and false-negative PET findings in cancer patients $[8,16]$. However, tissue sampling appears to be the only way to define the aetiology and clinical significance of focal areas of FDG uptake in the GIT in individual patients [17].

In the present study, PET/CT had a better sensitivity for the detection of iML in patients with proven malignancy. In the study by $\mathrm{CHOI}$ et al. [10], sensitivity and positive predictive value of PET/CT in detecting second primary cancer or unexpected
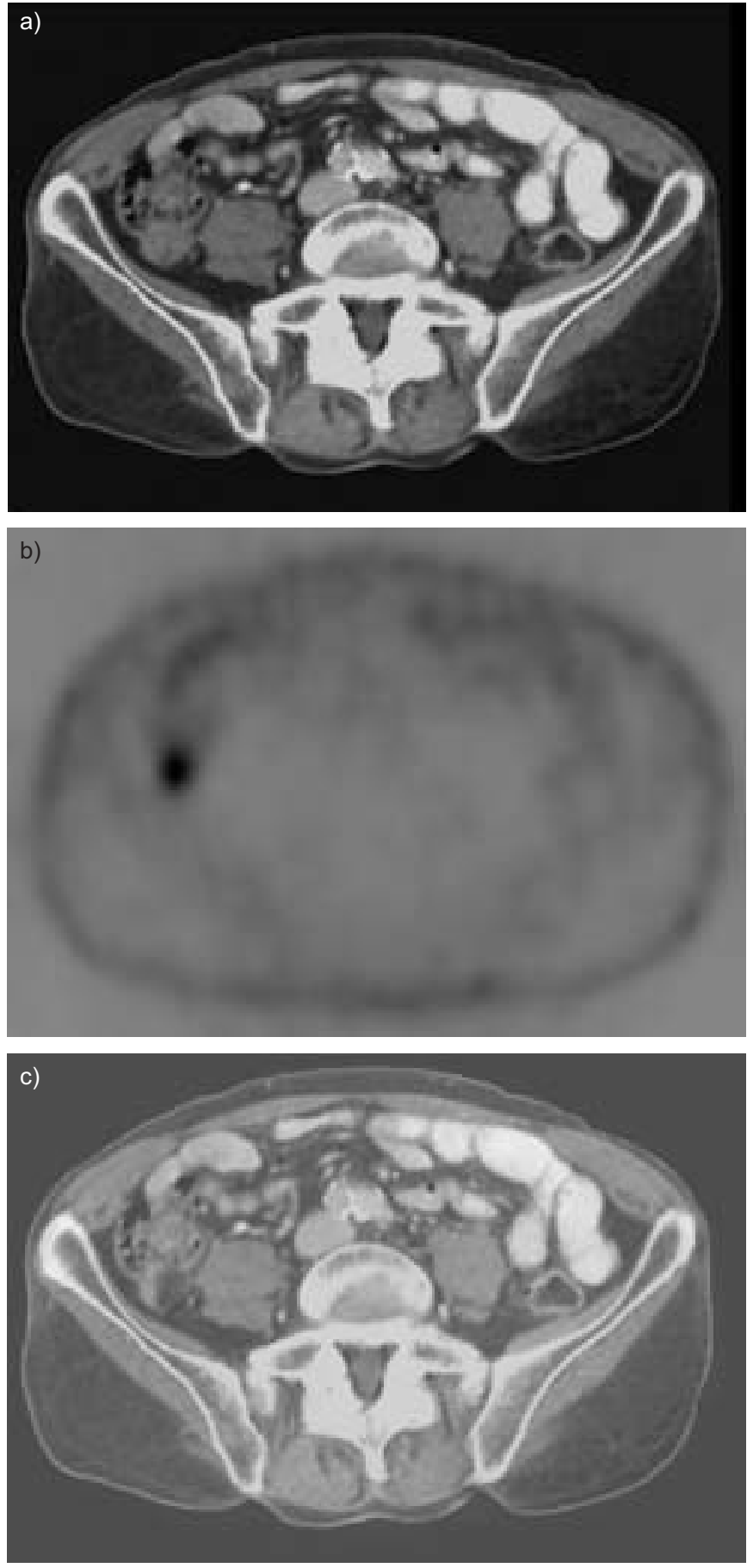

FIGURE 2. A 70-yr-old male with an adenocarcinoma in the right upper lobe a) The computed tomography (CT) part of the positron emission tomography (PET)/ CT demonstrates no additional extrapulmonary lesion. b) The PET part of the PET/ CT shows an F-fluoro-2-deoxyglucose (FDG) hotspot in the region of the right fossa iliaca suggestive of a malignant extrapulmonary lesion. c) PET/CT localised this FDG hotspot in the caecum, however, further investigation could not reveal a malignant lesion into the caecum. PET and PET/CT were false positive in this case.

metastasis of 91 and $69 \%$, respectively, were found; this was comparable with the results of the present study.

The present study has some limitations related to the techniques used and the selection of patients. The CT 
component of the PET/CT used in the present study is not dedicated to scanning of the chest, abdomen and skull, and a two-row spiral CT system was used. In current practice, conventional staging procedures are carried out using 16- or 64-multislice CT systems, which provide a better image quality; however, integrated PET/CT systems with incorporation of these dedicated multislices systems are presently rarely available. Taking into consideration the CT system used in the present study and the specific imaging technique of integrated $\mathrm{PET} / \mathrm{CT}$, the CT images evaluated have a lower image quality than could be expected if a standalone spiral CT or multislice $\mathrm{CT}$ were to be used. The CT part of the integrated PET/CT was performed with a lower dose ( 80 versus $120 \mathrm{~mA}$ for a dedicated chest CT in the Dept of Radiology, University Hospitals Gasthuisberg, Leuven, Belgium) [23]; however, HANY et al. [24] compared different $\mathrm{CT}$ doses and found no significant differences in accuracy when $80 \mathrm{~mA}$ was used compared with that of a dedicated chest CT. The CT was performed at normal expiratory breath-hold (limited breath-hold technique) versus at deep inspiration for a dedicated chest CT. This could induce problems in the correlation of lesions in the region of the diaphragm, especially lesions in the liver or colon. The CT examinations were performed with intravenous and oral contrast administration. The systematic use of intravenous contrast is considered necessary for a good interpretation of parenchymal changes on CT but could have caused problems during attenuation correction for PET imaging, although artefacts due to the use of intravenous CT contrast media are minimalised [25-27]. Conversely, the fact that no pre-contrast CT scans were performed has probably also negatively influenced the results of the CT scan, especially in the evaluation of the adrenal glands. The use of oral contrast agents can improve PET/CT specificity by a better delineation of intestinal structures, thus allowing a better discrimination between physiological and abnormal FDG uptake. An overestimation of the FDG activity measured on the attenuationcorrected PET images due to oral contrast agents has been shown [28]. However, this phenomenon does not seem to have much clinical effect. No significant difference in the distribution of intense FDG uptake in the intestinal regions was shown by DizENDORF et al. [29] in a study of two groups of 30 patients each. To minimise these artefacts, low-density barium appears to be suitable for clinical use [30]; administration of a negative oral contrast agent using mannitol is also promising [31].

Additionally, because CT is used for attenuation correction, PET images obtained from the integrated PET/CT are qualitatively better than "regular" FDG-PET images [8]. This can influence the interpretation of the PET images.

The present study reports CT, PET and PET/CT images without knowledge of the clinical follow-up of the patient. As the study was retrospective, it was impossible to evaluate the exact influence of the use of integrated PET/CT on the treatment strategy of the individual patient. However, through the number of patients included, the present study shows that integrated PET/CT has an important additional value in the staging of a lung tumour.

\section{Conclusions}

In the present study, positron emission tomography/computed tomography was demonstrated to depict more distant malignant extrapulmonary lesions than computed tomography and positron emission tomography alone. The introduction of positron emission tomography/computed tomography results in a computed tomography of the total abdomen and provides additional anatomical information of the lower abdomen. Combining metabolic and anatomical information, positron emission tomography/computed tomography has been advocated as a useful novel imaging tool leading to a decrease in the number of false-positive and false-negative positron emission tomography and computed tomography findings in cancer patients $[8,16]$. However, the precise localisation of increased F-fluoro-2-deoxyglucose foci using positron emission tomography/computed tomography cannot always solve the diagnostic dilemma of abnormal tracer uptake at present. A solitary F-fluoro-2-deoxyglucose accumulation that determines the possibility for radical treatment presently still requires a histopathological diagnosis [9].

\section{REFERENCES}

1 Verschakelen JA, De Wever W, Bogaert J. Role of computed tomography in lung cancer staging. Curr Opin Pulm Med 2004; 10: 248-255.

2 Saunders CA, Dussek JE, O'Doherty, et al. Evaluation of fluorine-18-fluorodeoxyglucose whole body positron emission tomography imaging in the staging of lung cancer. Ann Thorac Surg 1999; 212: 803-809.

3 Stroobants SG, D'Hoore I, Dooms C, et al. Additional value of whole-body fluorodeoxyglucose positron emission tomography in the detection of distant metastases of nonsmall-cell lung cancer. Clin Lung Cancer 2003; 4: 242-247.

4 Beyer T, Townsend DW, Brun T, et al. A combined PET/CT scanner for clinical oncology. J Nucl Med 2000; 41: 1369-1379.

5 De Wever W, Ceyssens S, Mortelmans L, et al. Additional value of PET-CT in the staging of lung cancer: comparison with CT alone, PET alone, and visual correlation of PET and CT. Eur Radiology 2007; 17: 23-32.

6 Cook GJ, Fogelman I, Maisey MN. Normal physiological and benign pathological variants of 18-fluoro-2deoxyglucose positron-emission tomography scanning: potential for error in interpretation. Semin Nucl Med 1996; 26: 308-314.

7 Osman MM, Cohade C, Fishman EK, et al. Clinically significant incidental findings on the unenhanced CT portion of PET/CT studies: frequency in 250 patients. J Nucl Med 2005; 46: 1352-1355.

8 Bar-Shalom R, Yefremov N, Guralnik L, et al. Clinical performance of PET/CT in evaluation of cancer: additional value for diagnostic imaging and patient management. J Nucl Med 2003; 44: 1200-1209.

9 Lardinois D, Weder W, Roudas M, et al. Etiology of solitary extra pulmonary positron emission tomography and computed tomography findings in patients with lung cancer. J Clin Oncol 2005; 23: 6846-6853.

10 Choi JY, Lee KS, Kwon OJ, et al. Improved detection of second primary cancer using integrated [18F]fluorodeoxyglucose positron emission tomography and computed tomography for initial tumor staging. J Clin Oncol 2005; 23: 7654-7659.

11 Ishimori T, Patel PV, Wahl RL. Detection of unexpected additional primary malignancies with PET/CT. J Nucl Med 2005; 46: 752-757. 
12 Yasuda S, Ide M, Fujii H, et al. Application of positron emission tomography imaging to cancer screening. $\mathrm{Br} \mathrm{J}$ Cancer 2000; 83: 1607-1611.

13 Dong C, Hemminki K. Second primary neoplasms among 53, 159 haematolymphoproliferative malignancy patients in Sweden, 1958-1996: a search for common mechanisms. Br J Cancer 2001; 85: 997-1005.

14 Ueno M, Muto T, Oya M, et al. Multiple primary cancer: an experience at the Cancer Institute Hospital with special reference to colorectal cancer. Int J Clin Oncol 2003; 8: 162-167.

15 Shen YY, Su CT, Chen GJ, et al. The value of ${ }^{18} \mathrm{~F}$-fluorodeoxyglucose positron emission tomography with the additional help of tumor markers in cancer screening. Neoplasma 2003; 50: 217-221.

16 Agress H Jr, Cooper BZ. Detection of clinically unexpected malignant and premalignant tumors with whole-body FDG PET: histopathologic comparison. Radiology 2004; 230: 417-422.

17 Israel $\mathrm{O}$, Yefremov $\mathrm{N}$, Bar-Shalom $\mathrm{R}$, et al. PET/CT detection of unexpected gastrointestinal foci of 18F-FDG uptake: incidence, localization patterns, and clinical significance. J Nucl Med 2005; 46: 758-762.

18 Rosenbaum SJ, Stergar H, Antoch G, Veit P, Bockisch A, Kuhl H. Staging and follow-up of gastrointestinal tumours with PET CT. Abdom Imaging 2006; 31: 25-35.

19 Bicik I, Bauerfeind $\mathrm{P}$, Breitbach $\mathrm{T}$, et al. Inflammatory bowel disease activity measured by positron-emission tomography. Lancet 1997; 350: 262.

20 Shreve PD, Anzai Y, Wahl RL. Pitfalls in oncologic diagnosis with FDG PET imaging: physiologic and benign variants. Radiographics 1999; 19: 61-77.

21 Gutman F, Alberini JL, Wartski M, et al. Incidental colonic focal lesions detected by FDG PET/CT. AJR Am J Roentgenol 2005; 185: 495-500.
22 Israel O, Mor M, Guralnik L, et al. Is ${ }^{18}$ F-FDG PET/CT useful for imaging and management of patients with suspected occult recurrence of cancer? J Nucl Med 2004; 45: 2045-2051.

23 Kamel E, Hany TF, Burger C, et al. CT vs. 68Ge attenuation correction in a combined PET/CT system: evaluation of the effect of lowering the CT tube current. Eur J Nucl Med Mol Imaging 2002; 29: 346-350.

24 Hany TF, Steinert HC, Goerres GW, et al. PET diagnostic accuracy: improvement with in-line PET-CT system: initial results. Radiology 2002; 225: 575-581.

25 Antoch G, Freudenberg LS, Beyer T, et al. To enhance or not to enhance? 18F-FDG and CT contrast agents in dual-modality 18F-FDG PET/CT. J Nucl Med 2004; 45: Suppl. 1, 56S-65S.

26 Yau YY, Chan WS, Tam YM, et al. Application of intravenous contrast in PET/CT: does it really introduce significant attenuation correction error? J Nucl Med 2005; 46: 283-291.

27 Dizendorf E, Hany TF, Buck A, et al. Cause and magnitude of the error induced by oral CT contrast agent in CT-based attenuation correction of PET emission studies. J Nucl Med 2003; 44: 732-738.

28 Antoch G, Freudenberg LS, Egelhof T, et al. Focal tracer uptake: a potential artefact in contrast-enhanced dualmodality PET/CT scans. J Nucl Med 2002; 43: 1339-1342.

29 Dizendorf EV, Treyer V, Von Schulthess GK, et al. Application of oral contrast media in coregistered positron emission tomography-CT. AJR Am J Roentgenol 2002; 179: 477-481.

30 Cohade C, Osman M, Nakamoto Y, et al. Initial experience with oral contrast in PET/CT: phantom and clinical studies. J Nucl Med 2003; 44: 412-416.

31 Antoch G, Kuehl H, Kanja J, et al. Dual-modality PET/CT scanning with negative oral contrast agent to avoid artefacts: introduction and evaluation. Radiology 2004; 230: 879-885. 